\title{
Accuracy Analysis of Positioning Method for Fusion GPS Pseudo-range and Continuous Wave Radar
}

\author{
Huijuan Zhang \\ Section 94, Unit 91550 \\ Dalian, China \\ Dinkey_s@163.com
}

\author{
Chao Wei \\ Section 94, Unit 91550 \\ Dalian, China \\ odinwell@163.com
}

\begin{abstract}
Continuous wave (CW) radar and global positioning system (GPS) is the main equipment of trajectory measurement in range. Based on range test background, propose a method to fuse GPS pseudo-range and $\mathrm{CW}$ radar range to provide complete trajectory positioning parameters, effectively reduce the impact of the systematic error of the measuring element to improve positioning precision of aircraft orbit solving. Through research on the measurement principle and the practice calculated data is given in this paper. Finally, several famous practical examples and simulation results are presented to illustrate our method efficiently.
\end{abstract}

Keywords- GPS pseudo-range; continuous wave ranging; data-fusion; accuracy analysis

\section{INTRODUCTION}

Continuous wave (CW) radar is a main equipment of trajectory measurement, but it often influenced by factors such as environment and status, sometimes it cannot continuous tracking of the target, or due to poor measurement precision, The precision of the trajectory cannot meet measurement requirements. At this time, we can add other measurement data to participate in track fusion solver to provide complete trajectory positioning parameters, effectively reduce the impact of the systematic error of the measuring element to improve positioning precision of aircraft orbit solving.

Global positioning system (GPS) has the characteristics of measuring the complete trajectory tracking, because it can be performed continuously, it meets a complete trajectory measurement conditions. At present, the exterior trajectory data processing only use trajectory results with other measurement devices measuring elements combined treatment, it does not give full play to the role of the GPS measurement data. The GPS pseudo-range was seen as the original measurement information with the $\mathrm{CW}$ radar ranging elements, using data fusion method for trajectory positional parameters. Through systematic error modeling and estimation, effectively reduce ranging error affects the precision of the results in order to obtain the high precision trajectory.

This article uses a variety of mark and parameter requirements are as follows: $m$ is the number of samples of the measuring element, $M$ is the number of stations, $N$ is the number of parameters to be estimated; the value of the $j$ measuring element is depicted in $y_{j i}=y_{j}\left(t_{i}\right)$ at time $t_{i}$, the signal to be measured is $f_{j}\left(t_{i}\right)$, the $j$ measuring element at every sampling time constitutes a vector is can be expressed as $Y_{j}=\left(y_{j}\left(t_{1}\right), \ldots, y_{j}\left(t_{m}\right)\right)^{T}$, the vector formed by the measurement data at time $t_{i}$ is expressed as $Y(i)=\left(Y_{1}\left(t_{i}\right), \ldots, Y_{M}\left(t_{i}\right)\right)^{T}$.

\section{MATHEMATICAL MODEL}

\section{A. Linear Representation of the Trajectory Parameters}

The trajectory parameters fusion solving was based primarily on spline function representation theory[1]. In the launch coordinates system, set GPS pseudo-range and CW radar measuring element trajectory parameters data fusion in three directions $x, y, z$ expressed as follows:

$$
\begin{cases}x(t)=\sum_{j=1}^{N} c_{j} \psi_{j}(t), & \dot{x}(t)=\sum_{j=1}^{N} c_{j} \dot{\psi}_{j}(t) \\ y(t)=\sum_{j=1}^{N} c_{j+N} \psi_{j}(t), \quad \dot{y}(t)=\sum_{j=1}^{N} c_{j+N} \dot{\psi}_{j}(t), \quad t_{1} \leq t \leq t_{m} \\ z(t)=\sum_{j=1}^{N} c_{j+2 N} \psi_{j}(t), \quad \dot{z}(t)=\sum_{j=1}^{N} c_{j+2 N} \dot{\psi}_{j}(t)\end{cases}
$$

Here is the trajectory linear model. In order to facilitate discussion, assume that $x, y, z$ three directions, the number of parameters to be estimated is equal. Wherein, $\left\{\psi_{1}(t), \psi_{2}(t), \ldots, \psi_{N}(t)\right\}$ is a known set of linearly independent spline basis functions, $C_{j}$ is the spline coefficients, when the value of $t_{m}-t_{1}$ is smaller, take a smaller value of $N$, And $\psi_{j}(t)$ is a low-order polynomial, and if the value of $t_{m}-t_{1}$ is larger, $\psi_{j}(t)$ can be taken as equidistant spline or unequal node B-spline.

\section{B. Nonlinear Model of the Real Signal}

In the launch coordinates system, ranging element and velocity measuring element of the GPS pseudo-range and CW radar are all nonlinear functions. As shown by (1). So ranging and velocimetry expression as follows (2):

In launch coordinate system, $(x(t), y(t), z(t), \dot{x}(t), \dot{y}(t), \dot{z}(t))$ is trajectory parameters at time $t$ and $\left(x_{k}, y_{k}, z_{k}\right)$ is the coordinate of the measurement station $k$.The error characteristics of the different measuring element is also different, data fusion can establish the real signal and measuring element error integration model. 


$$
\left\{\begin{aligned}
R_{k}(t)= & \sqrt{\left|x(t)-x_{k}\right|^{2}+\left|y(t)-y_{k}\right|^{2}+\left|z(t)-z_{k}\right|^{2}} \\
= & \sqrt{\left|\sum_{j=1}^{N} c_{j} \psi_{j}(t)-x_{k}\right|^{2}+\left|\sum_{j=1}^{N} c_{j+N} \psi_{j}(t)-y_{k}\right|^{2}} \\
& \dot{R}_{k}(t)=\frac{\left[x(t)-x_{k}\right] \dot{x}(t)+\left[y(t)-y_{k}\right] \dot{y}(t)+\left[z(t)-z_{k}\right] \dot{z}(t)}{R_{k}(t)} \\
= & \frac{1}{R_{k}(t)}\left\{\left(\sum_{j=1}^{N} c_{j} \psi_{j}(t)-x_{k}\right) \sum_{j=1}^{N} c_{j} \dot{\psi}_{j}(t)\right. \\
& +\left(\sum_{j=1}^{N} c_{j+N} \psi_{j}(t)-y_{k}\right) \sum_{j=1}^{N} c_{j+N} \dot{\psi}_{j}(t) \\
& \left.+\left(\sum_{j=1}^{N} c_{j+2 N} \psi_{j}(t)-z_{k}\right) \sum_{j=1}^{N} c_{j+2 N} \dot{\psi}_{j}(t)\right\}
\end{aligned}\right.
$$

\section{Measuring Element Integration Modeling}

let $C_{P}=\left\{c_{1}, c_{2}, \cdots, c_{N}, c_{N+1}, \cdots, c_{2 N}, c_{2 N+1}, \cdots, c_{3 N}\right\}$ By (1), (2), the trajectory parameter is linear function of the spline coefficients $C_{p}$ when given time ${ }^{t_{i}}$. GPS pseudo-range and $\mathrm{CW}$ radar ranging velocimetry real signal is a nonlinear function of $C_{p}$. All measuring element system error parameters to be estimated vector denoted by $a=\left(a_{1}, \cdots, a_{K}\right)^{\mathrm{T}}(0 \leq K<M)$. Thus the observed value of the measuring element of the respective times can be expressed as

$$
\begin{cases}\tilde{R}_{j}\left(t_{i}\right)=f_{j i}\left(C_{p}, a\right)+\varepsilon_{j}\left(t_{i}\right), & 1 \leq j \leq M_{1} \\ \tilde{R}_{j}\left(t_{i}\right)=f_{j i}\left(C_{p}, a\right)+\varepsilon_{j}\left(t_{i}\right), & M_{1}<j \leq M_{1}+M_{2} \\ \tilde{R}_{j}\left(t_{i}\right)=f_{j i}\left(C_{p}, a\right)+\varepsilon_{j}\left(t_{i}\right), & M_{1}+M_{2}<j \leq M_{1}+2 M_{2}\end{cases}
$$

As in (3), represents there are $M_{1}$ GPS satellites and $M_{2}$ sets of CW radar. Denoted $M=M_{1}+2 M_{2}, C=\left(C_{p}^{\mathrm{T}}, a^{\mathrm{T}}\right)^{\mathrm{T}}$,

$$
\begin{gathered}
F\left(t_{i}, C\right)=\left(\begin{array}{c}
f_{1 i}\left(C_{p}, a\right) \\
f_{2 i}\left(C_{p}, a\right) \\
\cdots \\
f_{M i}\left(C_{p}, a\right)
\end{array}\right), \quad \varepsilon(i)=\left(\begin{array}{c}
\varepsilon_{1}\left(t_{i}\right) \\
\varepsilon_{2}\left(t_{i}\right) \\
\cdots \\
\varepsilon_{M}\left(t_{i}\right)
\end{array}\right) \\
Y(i)=\left(\tilde{R}_{1}\left(t_{i}\right), \cdots, \tilde{R}_{M_{1}}\left(t_{i}\right), \cdots, \tilde{R}_{M_{1}+M_{2}}\left(t_{i}\right), \cdots, \tilde{\dot{R}}_{M}\left(t_{i}\right)\right)^{\mathrm{T}}
\end{gathered}
$$

Exterior ballistic measurement data fusion model at time $t_{i}$ is given as

$$
Y(i)=F\left(t_{i}, C\right)+\varepsilon(i)
$$

Used in combination the data of the time $t$ can be obtained equation

$$
Y=F(C)+\varepsilon
$$

$Y=\left(y_{1}, \cdots, y_{m}\right)^{\mathrm{T}}, C=\left(c_{1}, \cdots, c_{n}\right)^{\mathrm{T}}, F(C)=\left(f_{1}(C), \cdots, f_{m}(C)\right)^{\mathrm{T}}, \mathcal{E} \sim\left(0, \sigma^{2} I\right)$ As in (5), the trajectory parameters fusion processing GPS pseudo-range and the $\mathrm{CW}$ radar measuring element integration model. This is a non-linear model, its parameters to be estimated spline coefficients and the systematic error of the measuring element. Using non-linear regression method, the estimated value of the two can be obtained simultaneously. The model solution can use Gauss-Newton method [1].

\section{DATA FUSION}

\section{A. Processing Steps}

GPS pseudo-range and CW radar both are slant range information. GPS satellites can be treated as a station, data fusion in a uniform coordinate system for solving. The fusion process is presented as shown in Fig 1.

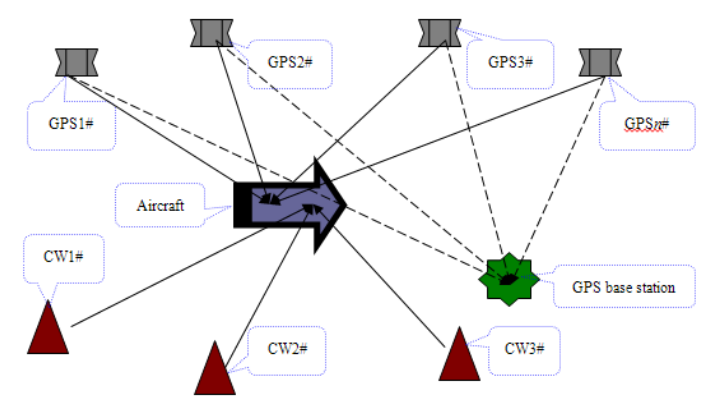

Figure 1. Data fusion schematic diagram

The data processing step are as follows:

Step 1: Convert the original binary file from receiver which accept from GPS base station and mobile station to RENIX format observation data files and navigation message [2].

Step 2: Extracted from the observational data file for the GPS satellite to the receiver pseudo-range.

Step 3: Obtain navigation files extracted from the GPS satellite ephemeris which is GPS satellite position every moment in the WGS-84 coordinate system.

Step 4: Select the appropriate GPS base stations to calculate the real distance of the satellite to the base station at various moments, and acceptable to the base station of the satellite pseudo-range differential obtain Pseudo-range correction amount.

Step 5: Using the correction amount that obtained in Step 4 corrected pseudo-range, in order to eliminate or weaken the receiver common errors and propagation delay error.

Step 6: Corrected pseudo-range and CW Ranging to have a series of processing, including tracking point inconsistency correction, data smoothing, measuring element matching check and random error statistics, etc[3].

Pay attention to the specific implementation process:

- Selection differential reference station is an iterative calculation process. According to the preliminary results determine the receiver and base station baseline length whether meet the pseudo-range differential conditions, if the result is not satisfactory you can select another recalculated.

- The pseudo-range differential technology can not eliminate the inherent errors such as internal noise, channel delay, multi-path effects, etc. It can be estimated in the process of fusion calculation analysis. 
- Consider the optimization station distribution method [4].

- The difficulty lies in time unifying in the differential process satellite ephemeris and the time of observation data [5].

\section{B. Accuracy Evaluation}

Positioning accuracy is assessed using the simulation method the following is specific process.

Step 1: A simulation trajectory is given as a reference trajectory. There are some measurement data from a calibration flight task. The GPS measured using the dualfrequency carrier receiver system. Waypoint software calculated positioning parameters is $X(t)$, theoretical precision less than $0.4 \mathrm{~m}$, the trajectory can be used as a reference trajectory.

Step 2: By the GPS pseudo-range and CW radar measuring element trajectory parameters fusion solving the trajectory position parameter $\hat{X}(t)$ and the fusion trajectory was reversed calculation of measuring element, denoted $\hat{Y}_{k}(t)$.

Step 3: Calculate a position error $\Delta X(t)=\hat{X}(t)-X(t)$ and the measuring element error $\Delta Y_{k}(t)=\hat{Y}_{k}(t)-Y_{k}(t) \cdot Y_{k}(t)$ is an original measuring element, $k$ is the number of measuring element.

Step 4: Statistical position and measuring element mean and standard deviation.

Typically, the standard deviation is a measure of random precision. In this paper the evaluation of the accuracy is measuring element residuals and standard deviation as the basis of the calculation and processing results.

\section{NUMERICAL EXAMPLE}

\section{A. Stations and Data}

Considering the effective data recording situation and station distribution, select three continuous wave radar stations for intersection, called CW2 \# CW3\# CW5\#. Base station and mobile station receiver outputs binary data files are converted navigation files and observation data files, extract the $\mathrm{P}$ code pseudo-range observables. By the Waypoint software calculate the dual frequency carrier relative positioning trajectory parameter [6], as shown in Fig 2. JZ1 is selected as a differential reference station. Bold line in the figure is the air route to be analysis.

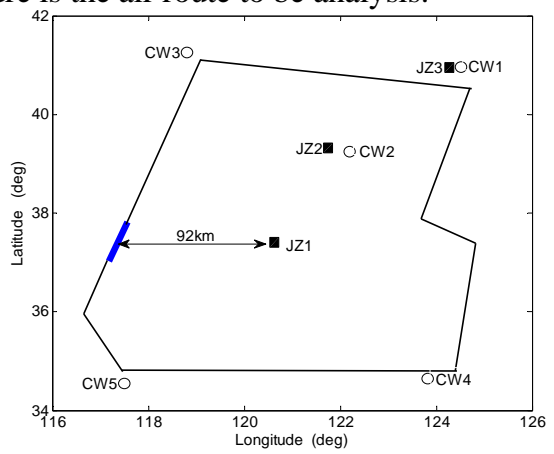

Figure 2. Air route and station distribution
Ephemeris from the satellite broadcast navigation message, which is the basic data of the position calculation. Satellite position can be calculated by the navigation data file.[7] Fusion data select GPS17 \# 24 \# two satellite pseudorange and the bomb bay observables.

Based on the previously discussion on the accuracy of the trajectory parameters estimation method. In this paper, the precision analysis and evaluation of the processing results based on the parameters residuals and root mean square error. Preprocessed measuring element and basis trajectory inverse calculations to the station measuring element residuals mean the root mean square error, as shown in Table I.

TABLE I. RANGING RESIDUALS MEAN AND RMS

\begin{tabular}{|c|c|c|}
\hline Station & Ranging mean(m) & Ranging Stdev(m) \\
\hline CW2\# & -75.222 & 0.376 \\
\hline CW3\# & -3.543 & 0.654 \\
\hline CW5\# & -0.827 & 1.014 \\
\hline GPS17\# & 5.974 & 1.724 \\
\hline GPS24\# & 28.199 & 1.189 \\
\hline
\end{tabular}

CW2\#,CW3\# station data containing systematic error, as shown in Figure 3.
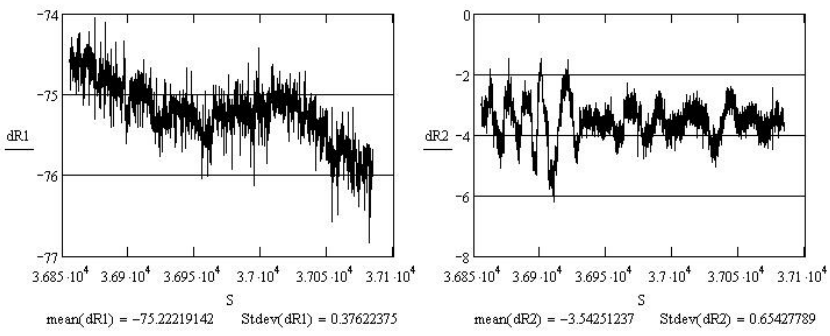

Figure 3. CW2\#,3\# Ranging residual result

The modified GPS pseudo-range still exists bigger system error, this is due to their inherent errors, and pseudorange differential method by the baseline length and the influence of satellite ephemeris is taken from the precision of the navigation message.

\section{B. Scheme 1}

Fusion data are only CW ranging. Because CW2\# station location using large constant errors, leading to three station intersection calculation of positioning accuracy is poor. Calculation results as shown in "Table II".

TABLE II. ESTIMATION RESULT FOR CW 3 STATIONS INTERSECTION

\begin{tabular}{|c|c|c|c|c|}
\hline Scheme & Param. & Mean(m) & RMS(m) & $\begin{array}{c}\text { Constant error } \\
\text { estimation(m) }\end{array}$ \\
\hline \multirow{2}{*}{$\begin{array}{c}\text { Without } \\
\text { constant } \\
\text { error } \\
\text { estimation }\end{array}$} & $\mathrm{X}$ & 34.1782 & 2.5329 & \\
\cline { 2 - 5 } & $\mathrm{Y}$ & -257.4006 & 59.8413 & \\
\cline { 2 - 5 } & $\mathrm{Z}$ & -77.5798 & 2.4369 & \\
\hline $\begin{array}{c}\text { CW2\# } \\
\text { constant } \\
\text { error } \\
\text { estimation }\end{array}$ & $\mathrm{X}$ & 216.4773 & 15.1141 & \\
\cline { 2 - 5 } & $\mathrm{Y}$ & -1487.3587 & 410.4717 & \\
\cline { 2 - 5 } & $\mathrm{Z}$ & -470.6622 & 12.7532 & 370.712 \\
\hline
\end{tabular}

From the statistical results, the intersection of three stations, some measuring element contains larger systematic error will pull partial whole trajectory, resulting in poor 
accuracy. At this time it is necessary to use other the effectively measuring element participate fusion.

\section{Scheme 2}

"TABLE III" shows the CW radar ranging without systematic error estimation. Error is estimated of fusion calculated CW radar and GPS five stations intersection positioning residuals.

TABLE III. ESTIMATION RESULT FOR 3 CW STATIONS INTERSECTION

\begin{tabular}{|c|c|c|}
\hline Param. & Mean(m) & RMS(m) \\
\hline X & -3.56 & 0.3062 \\
\hline Y & -6.948 & 1.979 \\
\hline Z & 1.147 & 0.7584 \\
\hline CW2\# R & -74.96 & 0.4865 \\
\hline CW3\# R & -0.1081 & 0.6441 \\
\hline CW5\# R & -2.325 & 1.057 \\
\hline GPS17\# R & -0.3851 & 0.6751 \\
\hline GPS24\# R & 22.68 & 0.918 \\
\hline
\end{tabular}

Positioning residuals curve in Fig 4(CW radar ranging without systematic error estimation).
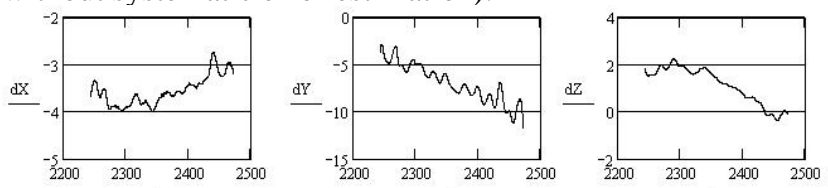

$\operatorname{mean}(d X)=-3.5598$ Stder $(d X)=0.3062 \operatorname{mean}(d Y)=-6.9484 \operatorname{Stdev}(d Y)=1.9787 \operatorname{mean}(d Z)=1.1469 \stackrel{S}{S} \operatorname{StdeV}(d Z)=0.7584$

Figure 4. 5 stations intersection positioning residual curve

"TABLE IV" shows the CW radar ranging systematic error estimation. Error is estimated of CW radar and GPS five stations intersection positioning residuals.[8]

TABLE IV. ESTIMATION RESULT FOR 5 CW STATIONS INTERSECTION

\begin{tabular}{|c|c|c|c|}
\hline Param. & Mean(m) & RMS(m) & $\begin{array}{c}\text { Constant error } \\
\text { estimation(m) }\end{array}$ \\
\hline X & -0.425 & 0.1519 & \\
\hline Y & -4.445 & 1.872 & \\
\hline Z & 1.303 & 0.171 & \\
\hline CW2\# R & -0.0006419 & 0.3694 & -76.150 \\
\hline CW3\# R & $-7.40 E-05$ & 0.6427 & -3.301 \\
\hline CW5\# R & 0.0001068 & 1.003 & \\
\hline GPS17\# R & -0.002525 & 0.6714 & 1.515 \\
\hline GPS24\# R & -0.03921 & 0.8504 & 24.079 \\
\hline
\end{tabular}

Positioning residuals curve in Fig 5(CW radar ranging systematic error estimation).
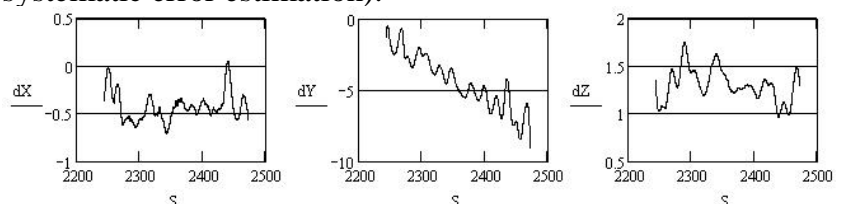

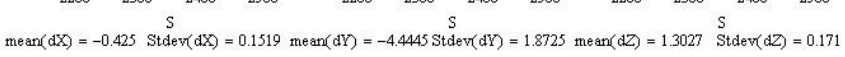

Figure 5. 5 stations intersection positioning residual result

Considering the CW2\# station location contains obvious systematic errors, the following solution only using CW3\#, 5\# and GPS17\# pseudo-range fusion positioning solving. Positioning residual as shown in "TABLE V", the result is estimated CW radar ranging systematic error.

TABLE V. ESTIMATION RESULT FOR 3 CW STATIONS INTERSECTION

\begin{tabular}{|c|c|c|c|}
\hline Param. & Mean(m) & RMS(m) & $\begin{array}{c}\text { Constant error } \\
\text { estimation(m) }\end{array}$ \\
\hline X & -1.365 & 0.3294 & \\
\hline Y & -4.794 & 1.836 & \\
\hline Z & 2.516 & 0.5249 & \\
\hline CW3\# R & $-3.42 \mathrm{E}-05$ & 0.6438 & -2.780 \\
\hline CW5\# R & $-3.20 \mathrm{E}-05$ & 1.002 & \\
\hline GPS17\# R & -0.0008323 & 0.6911 & 1.136 \\
\hline
\end{tabular}

Positioning residuals curve in Fig 6(CW radar ranging systematic error estimation).
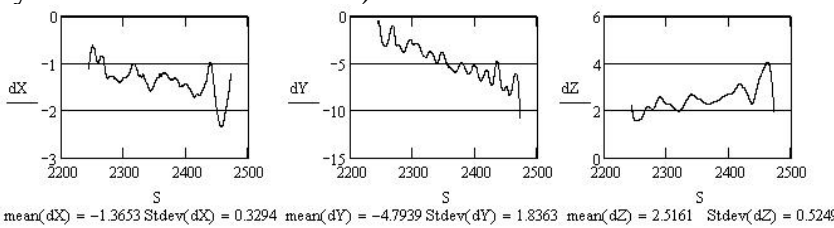

Figure 6. 3 stations intersection positioning residual result

\section{Analysis of Results}

When the CW measuring element is not enough or low accuracy, GPS effective pseudo-distance can be accurately estimated CW ranging system error, and can greatly improve the accuracy of the trajectory Fusion solving positioning results. The series schemes also verify this conclusion: when the GPS pseudo-range system error within a certain range, can be used as the necessary complement of the CW measuring element, to participate in fusion solving. Verified the contents of this study have practical value in the test.

\section{CONCLUSIONS}

The study shows that, to ensure accuracy and establish an appropriate mathematical model for the error correction, the GPS pseudo-range can be used to precise positioning. This paper establishes the GPS pseudo-range and CW radar measuring element data fusion system, realization of the satellite position calculation and pseudo-range extraction, And GPS satellite as a mobile station fusion algorithm, can be applied to tracking data processing in range.

\section{REFERENCES}

[1] W. Zhengming, Tracking data calibration and evaluation, Changsha: National University of Defense Technology press, 1999.

[2] W Gurtner. RINEX: The Receiver Independent Exchange Format Version 2.12. Canada : Astronomical Institute University of Berne,June 2009.

[3] J. Xingquan, Continuous-wave radar data processing, Beijing: Industrial National Defense Press, 2005.

[4] M. Shunnan and W. Wei, "Research on optimization station distribution method for exterior ballistic measuring system in range", Journal of Astronautics, 2008,06:1951-1954.

[5] Z. Shouxin, GPS satellite measurements positioning theory and application, Changsha: National University of Defense Technology press, 1996.

[6] C. Yi. L. Ying and N. Lei, "The application of carrier phase smoothing filter on gps pseudorange”, Aerospace control, vol. 01, 2011, pp:54-58.

[7] L. Hongtao, GPS application design, Beijing: Science press, 2000.

[8] W. Zhengming, "Estimation of constant systematic errors of continuous wave radar system" Chinese space science and technology, vol. 01, 1996, 11-19. 